\title{
PROBABILISTIC METHODS FOR DECOMPOSITION DIMENSION OF GRAPHS
}

\author{
Mariko Hagita, André Kündgen, and Douglas B. West
}

last revised February 4, 2003

\begin{abstract}
In a graph $G$, the distance from an edge $e$ to a set $F \subseteq E(G)$ is the vertex distance from $e$ to $F$ in the line graph $L(G)$. For a decomposition of $E(G)$ into $k$ sets, the distance vector of $e$ is the $k$-tuple of distances from $e$ to these sets. The decomposition dimension $\operatorname{dec}(G)$ of $G$ is the smallest $k$ such that $G$ has a decomposition into $k$ sets so that the distance vectors of the edges are distinct.

For the complete graph $K_{n}$ and the $k$-dimensional hypercube $Q_{k}$, we prove $(2-$ $o(1)) \lg n \leq \operatorname{dec}\left(K_{n}\right) \leq(3.2+o(1)) \lg n$ and $k / \lg k \leq \operatorname{dec}\left(Q_{k}\right) \leq(3.17+o(1)) k / \lg k$. The upper bounds use probabilistic methods directly or indirectly. We also prove that random graphs with edge probability $p$ such that $p n^{1-\varepsilon} \rightarrow \infty$ for some positive constant $\varepsilon$ have decomposition dimension $\Theta(\ln n)$ with high probability.
\end{abstract}

AMS classifications: 05C12, 05C35, 05D05, 05D40

Keywords: union-free family, probabilistic method, complete graph, hypercube, random graph, cartesian product

\section{INTRODUCTION}

The distance between edges $e$ and $f$ in a graph $G$, denoted by $d(e, f)$, is the distance between them as vertices in the line graph of $G$. Given an edge-coloring of $G$, the distance $d(e, c)$ between an edge $e$ and a color $c$ is the minimum distance from $e$ to edges of color $c$. Thus $d(e, c)=0$ if and only if $e$ has color $c$. We associate with each edge $e$ the distance vector $r(e)=\left(d\left(e, c_{1}\right), d\left(e, c_{2}\right), \ldots, d\left(e, c_{k}\right)\right)$.

Our edge-colorings are arbitrary partitions of $E(G)$, so we use the language of graph decomposition. A decomposition is distinguishing if the resulting distance vectors for edges are distinct. Chartrand, Erwin, Raines, and Zhang [2] defined the decomposition dimension $\operatorname{dec}(G)$ to be the minimum number of colors in a distinguishing decomposition of $G$. Since every distance vector has exactly one 0 and the remaining entries are at most $d+1$ when $G$ has diameter $d$, they observed that

$$
\operatorname{dec}(G)=k \quad \text { implies }|E(G)| \leq k(d+1)^{k-1} .
$$

This yields roughly $\operatorname{dec}(G)>\log _{d+1} m-\log _{d+1} \log _{d+1} m$ when $G$ has $m$ edges and diameter $d$. This bound can be weak: for a $\operatorname{star}, \operatorname{dec}\left(K_{1, n-1}\right)=n-1$. Enomoto [4] proved that $\operatorname{dec}(G) \leq n-1$ for every $n$-vertex graph $G$ except complete graphs with 3 or 4 vertices. Enomoto and Nakamigawa [5] proved another lower bound in terms of the maximum degree $\Delta(G)$ :

$$
\operatorname{dec}(G) \geq\lceil\lg \Delta(G)\rceil+1
$$


where $\lg$ denotes $\log _{2}$ (we also use $\ln$ for $\log _{e}$ ). They proved that for every $r \geq 1$ and $k \geq 3$ there is a tree $T$ with $\Delta(T)=k$ and $\operatorname{dec}(T)=\lceil\lg k\rceil+r$. For graphs with small diameter, the bound in (1) is sharper, and we will study graphs with this property. We say that a family $\mathcal{F}$ of graphs is well-behaved for decomposition dimension if there is a constant $c$ such that for all $G \in \mathcal{F}$, the decomposition dimension of $G$ is at most $c$ times the lower bound arising from (1); in particular, $\operatorname{dec}(G) \leq c \log _{d+1} m$, where $d$ and $m$ are the diameter and the number of edges of $G$. It would be interesting to find structural conditions for families to be wellbehaved. The family of stars is not well-behaved, but in this paper we show that the families of complete graphs, hypercubes, and random graphs are well-behaved, and we explore the question for cartesian products of identical complete graphs.

These results suggest that regularity or symmetry could be relevant structural properties. Indeed, the lower bound in (2) implies that a family cannot be wellbehaved if $\lg \Delta(G) \lg \operatorname{diam}(G) / \lg e(G)$ is unbounded for graphs in the family, where $e(G)$ and $\operatorname{diam}(G)$ denote the number of edges and the diameter of a graph $G$, respectively. The family of stars shows that boundedness of this ratio is not sufficient to make a family well-behaved.

Before proving our results, we note that decomposition dimension behaves well in another way. At the 14th Cumberland Conference (Memphis, 2001), D. Slilaty asked whether there is a distinguishing decomposition of $G$ into exactly $k$ subgraphs whenever $\operatorname{dec}(G) \leq k \leq e(G)$. Y. Egawa gave the following simple proof by successively splitting colors. Given a distinguishing decomposition with $k$ colors, split a color class $F$ into classes $F^{\prime}$ and $F^{\prime \prime}$. The distances from edges $e$ and $e^{\prime}$ to other colors don't change, so $r(e)$ and $r\left(e^{\prime}\right)$ remain distinct unless $e$ and $e^{\prime}$ have the same distance to $F^{\prime}$ and $F^{\prime \prime}$ but different distances to $F$. By symmetry, we may assume that $d(e, F)<d\left(e^{\prime}, F\right)$. The computation below now implies that $e$ and $e^{\prime}$ cannot have the same distances to both $F^{\prime}$ and $F^{\prime \prime}$.

$$
\min \left\{d\left(e^{\prime}, F^{\prime}\right), d\left(e^{\prime}, F^{\prime \prime}\right)\right\}=d\left(e^{\prime}, F\right)>d(e, F)=\min \left\{d\left(e, F^{\prime}\right), d\left(e, F^{\prime \prime}\right)\right\}
$$

\section{Complete GRAPhS AND Union-Free FAMilies of SETS}

For the complete graph $K_{n}$, the trivial lower bound resulting from (1) is about $2 \lg n-\lg \lg n$. In [2], it was proved that $\operatorname{dec}\left(K_{n}\right) \leq(2 n+5) / 3$ for $n \geq 3$. This bound was improved by Nakamigawa [8] to $\operatorname{dec}\left(K_{n}\right) \leq(1 / 2+o(1)) \lg ^{2} n$. In this paper we improve the upper bound to $(3.2+o(1)) \lg n$ using a construction based on union-free families of sets. Thus complete graphs are well-behaved and $\operatorname{dec}\left(K_{n}\right)=\Theta(\lg n)$.

Let $[k]=\{1, \ldots, k\}$. A family $\mathcal{F}$ of subsets of $[k]$ is strongly union-free if $A \cup B=$ $C \cup D$ implies $\{A, B\}=\{C, D\}$ whenever $A, B, C, D \in \mathcal{F}$ (not necessarily distinct). A family $\mathcal{F}$ is simple if $|\mathcal{F}|>1$ and $|B-A| \geq 2$ for all distinct $A, B \in \mathcal{F}$. Note that existence of a simple family requires $k \geq 4$.

Using the probabilistic method, Coppersmith and Shearer [3] proved the existence of a large family of subsets of $[k]$ that is both strongly union-free and simple. When $k$ is sufficiently large, the family has size $2^{(0.31349+o(1)) k}$; call this $n$. We use this family to show that $\operatorname{dec}\left(K_{n}\right) \leq k+1$. Solving for $n$ in terms of $k$ yields the bound we have claimed: $\operatorname{dec}\left(K_{n}\right) \leq(3.19+o(1)) \lg n$. The upper bound thus follows from our first result. 
Theorem 2.1. Let $G$ be an n-vertex graph containing the complete bipartite graph $K_{k, n-k}$ as a spanning subgraph. If there is a family consisting of $n-k$ subsets of $[k]$ that is both simple and strongly union-free, then $\operatorname{dec}(G) \leq k+1$.

Proof. Let $\mathcal{F}$ be such a family of subsets of $[k]$. We may assume that $\bigcup \mathcal{F}=[k]$, since uncovered elements may be added to any set in $\mathcal{F}$. We may also assume that every $i \in[k]$ is missing from at least one set in $\mathcal{F}$, since otherwise we may remove $i$ from some set. Since $\mathcal{F}$ is simple, every member of $\mathcal{F}$ has size at least 2 . From $\mathcal{F}$ we form a new family $\mathcal{F}_{0}$ by appending the element 0 to every set in $\mathcal{F}$ and adding the 2-element sets $\{0, i\}$ for $1 \leq i \leq k$.

Let $U$ and $W$ be the partite sets of a copy of $K_{k, n-k}$ in $G$, with $U=\left\{u_{1}, \ldots, u_{k}\right\}$. To each $v \in V(G)$, we assign a set $S_{v} \in \mathcal{F}_{0}$. Let $S_{u_{i}}=\{0, i\}$, and assign the remaining members of $\mathcal{F}_{0}$ to distinct vertices of $W$.

Next we color $E(G)$ by setting $c(u v)=\max \left(S_{u} \cap S_{v}\right)$ for each edge $u v$. This is well-defined, since each set contains 0 . By construction, every color incident to vertex $v$ belongs to $S_{v}$. Conversely, every element of $S_{v}$ is a color incident to $v$. When $S_{v}=\{0, i\}$, this holds because $i$ appears in some but not all elements of $\mathcal{F}$. When $v \in W$ and $0<i \in S_{v}$, color $i$ appears on the edge $u_{i} v$, while color 0 appears on each $u_{j} v$ such that $j \notin S_{v}$.

We claim that the resulting distance vectors are distinct.

The coordinate positions $[k] \cup\{0\}$ correspond to the colors. Since $S_{w}$ is the set of colors appearing at $w$, the distance vector $r(u v)$ is at most 1 in position $i$ if and only if $i \in S_{u} \cup S_{v}$. Hence $S_{u} \cup S_{v}$ is the set of positions where $r(u v)$ is at most 1. Let $S_{u v}=S_{u} \cup S_{v}$. To prove that $c$ produces distinct distance vectors, it now suffices to prove that $c(u v) \neq c(x y)$ when $S_{u v}=S_{x y}$ and $u v \neq x y$.

The color on $u v$ is the position of the unique 0 in $r(u v)$. When $u, v \in U$, we have $\left|S_{u v}\right|=3$ and $c(u v)=0$. Furthermore, for $u, v \in U$ the triples $S_{u v}$ are distinct. It is possible to have $\left|S_{u v}\right|=3$ when $u \in U$ and $v \in W$, but then $c(u v) \neq 0$. When $x, y \in W$, the sets of the form $S_{x} \cup S_{y}$ again are distinct, since $\mathcal{F}$ is strongly union-free, and they have size at least 5 , since $\mathcal{F}$ is simple.

Thus if $u v$ and $x y$ are distinct edges and $r(u v)=r(x y)$, we may assume that $u \in U$, that $v, y \in W$, and that $S_{u v}=S_{x y}$. Now $\left|S_{y} \cup S_{v}\right| \leq\left|S_{u v}\right| \leq\left|S_{v}\right|+1$ and simplicity imply that $S_{v}=S_{y}$ and thus also $x \in U$. If $c(u v)=i \neq 0$, then $S_{u}=\{0, i\}=S_{x}$, since otherwise $c(x y) \neq i$. On the other hand, $c(u v)=c(x y)=0$ implies that $S_{u v}=S_{v} \cup\{i\}$ and $S_{x y}=S_{y} \cup\{j\}$. Since $v=y$ and $S_{u v}=S_{x y}$, we conclude that $i=j$ and $u=x$.

The proof of Theorem 2.1 was motivated by a proof by Harary and Plantholt [6] that $\lceil\lg n\rceil+1$ is the minimum number of colors in an edge-coloring of $K_{n}$ such that the sets of colors appearing at vertices are distinct.

It is not necessary to check that the family generated by Coppersmith and Shearer is simple in order to obtain our bound $\operatorname{dec}\left(K_{n}\right) \leq(3.19+o(1)) \lg n$. Every strongly union-free family $\mathcal{F}^{*}$ has a large subfamily $\mathcal{F}$ that is simple. First pick $s$ such that the collection of $s$-sets in $\mathcal{F}^{*}$ has maximum size. Next, iteratively choose an $s$-set $S \in \mathcal{F}^{*}$ and discard the subsets that differ in only one element from $S$. Since there are at most $k(s-k)$ subsets discarded for each element chosen, we obtain $|\mathcal{F}| \geq\left|\mathcal{F}^{*}\right| /(k(1+s(k-s))) \geq 4\left|\mathcal{F}^{*}\right| / k^{3}$.

Our result does not completely settle the asymptotic question for $K_{n}$.

Question: Is it true for every positive real number $\varepsilon$ that $\operatorname{dec}\left(K_{n}\right) \leq(2+\varepsilon) \lg n$ when $n$ is sufficiently large? 


\section{A GENERAL UPPER BOUND}

The construction of Coppersmith and Shearer is essentially probabilistic. Our next upper bound on $\operatorname{dec}(G)$ is proved by applying the probabilistic method more directly. This is a structural upper bound for fixed graphs, proved by producing a decomposition probabilistically.

Theorem 3.1. Fix integers $d, s, k$ such that $d \geq s+1 \geq 3$ and $k \geq 5 \frac{d}{s} \lg e(G)+2$. If a graph $G$ contains $k$ pairwise edge-disjoint spanning subgraphs whose vertices all have degree between $s+1$ and $d$, then $\operatorname{dec}(G) \leq k+1$.

Proof. Let $G_{1}, \ldots, G_{k}$ be such a family of subgraphs. Let $t=d / s$, and define $p$ by $(1-p)^{s}=\frac{2 t}{2 t+1}$. Color the edges in $G$ so that each edge in $G_{i}$ has color $i$ with probability $p$ and has color 0 otherwise, and all other edges have color 0 . At most $k+1$ colors are used.

Consider two edges $e$ and $e^{\prime}$. If $e, e^{\prime} \notin E\left(G_{i}\right)$, then the probability that color $i$ distinguishes $e$ and $e^{\prime}$ is at least the probability that exactly one of $e$ and $e^{\prime}$ is incident to an edge of color $i$. At most $2 d$ edges of $G_{i}$ are incident to $e$. The number of edges of $G_{i}$ incident to $e^{\prime}$ but not to $e$ is at least $s$ if $e$ is incident to $e^{\prime}$. It is at least $2(s+1)-4$ if $e$ is not incident to $e^{\prime}$, since $e^{\prime} \notin E\left(G_{i}\right)$ implies that at least $2(s+1)$ edges of $G_{i}$ are incident to $e^{\prime}$, and at most four of these are also incident to $e$. The inequality $2(s+1)-4 \geq s$ reduces to $s \geq 2$, so we always have at least $s$ edges incident to $e^{\prime}$ but not to $e$.

Hence when $e, e^{\prime} \notin E\left(G_{i}\right)$ the probability that color $i$ is incident to $e^{\prime}$ but not to $e$ is at least $(1-p)^{2 d}\left(1-(1-p)^{s}\right)$. We can also switch the roles of $e$ and $e^{\prime}$. In the computation below, the last inequality follows from $t>1$.

$$
2(1-p)^{2 d}\left(1-(1-p)^{s}\right)=2\left(\frac{2 t}{2 t+1}\right)^{2 t} \frac{1}{2 t+1}=\left(1-\frac{1}{2 t+1}\right)^{2 t+1} \frac{1}{t}>\frac{0.296}{t} .
$$

Let $c=0.296 / t$. Since we have ignored the colors of $e$ and $e^{\prime}$ and the exceptional color 0 (one of these also may distinguish $e$ and $e^{\prime}$ ), we conclude that the probability that $e$ and $e^{\prime}$ are distinguished by no color is less than $(1-c)^{k-2}$.

There are $\left(\begin{array}{c}e(G) \\ 2\end{array}\right)$ pairs of edges. We obtain the existence of a distinguishing decomposition with at most $k+1$ colors if $(1-c)^{k-2} e(G)^{2} \leq 1$, or equivalently $k \geq 2-\frac{2}{\lg (1-c)} \lg e(G)$. Now it suffices to observe that $\ln (1-c) \leq-c \leq-0.296 / t$, so $-\frac{2}{\lg (1-c)}=-\frac{2 \ln 2}{\ln (1-c)} \leq \frac{2 \ln 2}{0.296} t<5 t$.

Corollary 3.2. If $G$ is an $n$-vertex $r$-regular graph with $r \geq 80 \lg n$, then $\operatorname{dec}(G) \leq$ $10 \lg (n r)$.

Proof. Let $d=4, s=2$, and $k=10 \lg (n r)-1$. Since $G$ has $n r / 2$ edges, it follows that $5 \frac{d}{s} \lg e(G)+1=10(\lg (n r)-1)+1<k$. Thus it suffices to find $k$ edge-disjoint spanning subgraphs in which all vertices have degree 3 or 4 .

If $r$ is even, then $G$ decomposes into 2-factors, by Petersen's Theorem [9]. Combining the 2-factors in pairs yields 4-regular spanning subgraphs. We only need $10 \lg (n r)-1$ such subgraphs, so $r \geq 80 \lg n$ is sufficient.

If $r$ is odd, then form a graph $G^{\prime}$ by taking two copies of $G$ and adding a matching that joins corresponding vertices in the two copies. Now $G^{\prime}$ is regular of degree $r+1$ and decomposes into 2-factors. Again these combine to form $k 4$ factors. Restricting these subgraphs to $G$ yields spanning subgraphs of $G$ in which 
every vertex has degree 3 or 4 , since each vertex has exactly one incident edge in $G^{\prime}$ that is not in $G$.

Corollary 3.3. Regular graphs of bounded diameter are well-behaved.

Proof. If the diameter of an $r$-regular graph on $n$ vertices is bounded by $d$, then $r^{d} \geq n$. This yields $r \geq n^{1 / d} \geq 80 \lg n$. Now Corollary 3.2 provides an upper bound on $\operatorname{dec}(G)$. To show that the family is well-behaved we show that this bound is at most a constant times the lower bound arising from (1).

$$
\begin{aligned}
\operatorname{dec}(G) & \leq 10 \lg (n r) \leq 20 \lg (e(G)) \\
& =20 \lg (d+1) \log _{d+1}(e(G)) \leq c_{d} \log _{1+\operatorname{diam} G}(e(G)) .
\end{aligned}
$$

\section{RANDOM GRAPHS}

Next we apply Theorem 3.1 to random graphs. We consider the model in which graphs with a specified vertex set are generated by letting each vertex pair be an edge with probability $p$, independently. The edge probability $p$ is a function of the number of vertices, $n$. A property holds "almost always" or "for almost every graph" in this model if its probability in the model with a specified vertex set of size $n$ tends to 1 as $n$ tends to $\infty$. Theorem 3.1 is used to obtain the upper bound, but also we will need results on the diameter of graphs generated with edge probability $p$ to make a precise statement about random graphs being well-behaved.

Theorem 4.1. If $p n / \ln ^{2} n \rightarrow \infty$, then in the random graph model with edge probability $p$ the inequality $\operatorname{dec}(G) \leq 20 \ln |V(G)|$ holds for almost every graph $G$.

Proof. We create simultaneously a random $n$-vertex graph $G$ and an edge decomposition $G_{1}, \ldots, G_{k}$, where $k=20 \ln n$. Each pair of vertices becomes an edge of $G$ with probability $p$. If a pair becomes an edge, then we place it into one of the subgraphs of the decomposition uniformly at random. Hence each pair has probability $p / k$ of becoming an edge in $G_{i}$, for $1 \leq i \leq k$.

Let $d_{i}(v)$ denote the degree of vertex $v$ in $G_{i}$. The random variable $d_{i}(v)$ is binomial random variable with $n-1$ trials and success probability $p^{\prime}$ equal to $p / k$. Its expectation $\lambda$ is $(n-1) p^{\prime}$.

Let $t=\sqrt{n p}$. We show that with high probability every vertex degree lies between $s+1$ and $d$, where $s+1=\lambda-t$ and $d=\lambda+t$ (note that $s+1 \geq 3$ for large $n$ ). This follows from Chernoff's Inequality bounding the tail probabilities in the binomial distribution (see, for example, page 26 of [7]). The probability that $d_{i}(v) \leq \lambda-t$ is bounded by $e^{-t^{2} /(2 \lambda)}$, and the probability that $d_{i}(v) \geq \lambda+t$ is bounded by $e^{-t^{2} /(2 \lambda+t)}$. We compute $e^{-t^{2} /(2 \lambda)} \leq e^{-k / 2} \leq n^{-10}$ and $e^{-t^{2} /(2 \lambda+t)} \leq$ $e^{-k / 2.5} \leq n^{-8}$.

The probability that some $d_{i}(v)$ is not between $s+1$ and $d$ is thus bounded by $k n\left(n^{-10}+n^{-8}\right)$, which tends to 0 . Since $e(G) \leq\left(\begin{array}{c}n \\ 2\end{array}\right)$, we have $\lg e(G) \leq 2 \lg n$. With $d$ and $s$ defined as above, this yields $5 \frac{d}{s} \lg e(G)+2 \leq 5(1+o(1)) 2 \lg n+2 \leq$ $20 \ln n=k$. Hence Theorem 3.1 applies almost always and completes the proof.

In considering whether random graphs generated with edge probability $p$ are wellbehaved, we need the behavior of $e(G)$ and $\operatorname{diam} G$ in this model. If the number of 
edges is almost always at least a constant positive power of $n$, and the diameter is almost always bounded by a constant, then there is a constant $c$ such that the ratio of the upper bound in Theorem 4.1 to the lower bound from (1) is almost always bounded by $c$. This fact about the ratio is precisely what we mean by "random graphs are well-behaved".

Corollary 4.2. For random graphs generated with edge probability $p$ such that $p n^{1-\varepsilon} \rightarrow \infty$ for some positive constant $\varepsilon$, there is a constant $c$ such that the probability of $\operatorname{dec}(G)$ being bounded by $c \log _{1+\operatorname{diam} G}(e(G))$ tends to 1 .

Proof. Let $N=\left(\begin{array}{l}n \\ 2\end{array}\right)$. When $p N \rightarrow \infty$, the number of edges in the random graph has a binomial distribution. It has expected value $p N$, and $e(G)$ is almost always between $(1-\varepsilon) p N$ and $(1+\varepsilon) p N$. When $p n^{1-\varepsilon} \rightarrow \infty$, almost always $e(G)$ exceeds $n^{1+\varepsilon}$.

Next consider the diameter. Let $D=\lceil 1 / \varepsilon\rceil$. Let $f(n)=n^{1-1 / D} /(\ln n)^{1 / D}$. Since $1-1 / D \geq 1-\varepsilon, p n^{1-\varepsilon} \rightarrow \infty$ implies that $p f(n) \rightarrow \infty$. Since $f(n)$ is a threshold for having diameter at most $D$ (Theorem 10 of Chapter 10 of [1]), we conclude that in the model with edge probability $p$, almost every graph has diameter at most $D$.

Now the lower bound of $(1)$ is almost always at least $(1-\varepsilon) \log _{1+D} e(G)$, which is almost always at least $\left(1-\varepsilon^{2}\right) \log _{1+D} n$. Since $p n^{1-\varepsilon} \rightarrow \infty$, also $p n / \ln ^{2} n \rightarrow \infty$. Hence Theorem 4.1 applies and yields $\operatorname{dec}(G) \leq 20 \ln n$ almost always. Thus $\operatorname{dec}(G)$ is almost always at most $c$ times the lower bound from (1), where $c=20 \ln (1+$ $\lceil 1 / \varepsilon\rceil) /\left(1-\varepsilon^{2}\right)$.

\section{Hypercubes}

We have studied graphs with bounded diameter. To show that the random method can also work for sparser graphs, we consider the hypercube.

The hypercube $Q_{k}$ is the graph with vertex set $\{0,1\}^{k}$ and edge set consisting of the pairs of $k$-tuples differing in one position. We write the edges as $k$-tuples with $\mathrm{a} *$ in the position where the endpoints differ; thus $001 * 0$ is the edge with endpoints 00100 and 00110 . The distance between two distinct edges is one more than the number of positions where one $k$-tuple has 0 and the other has 1 . We use the word "position" when discussing entries in the vectors for vertices and edges of $Q_{k}$ because we use the word "coordinate" when discussing entries in the distance vectors that arise from an edge-coloring.

Since the hypercube has diameter $k$ and $k 2^{k-1}$ edges, the simple lower bound from (1) implies that $\operatorname{dec}\left(Q_{k}\right)>k / \lg k$. We prove that this is sharp within a factor of 3.2. (A referee provided a simple explicit construction to show that $\operatorname{dec}\left(Q_{k}\right) \leq$ $k+1$; a random construction gives the right order of magnitude.)

Theorem 5.1. For $k$ sufficiently large, $\operatorname{dec}\left(Q_{k}\right) \leq 3.17 k / \lg k$.

Proof. We pick a set $C$ of $c=\lfloor 3.17 k / \lg k\rfloor-1$ edges at random and give them distinct colors; the remaining edges receive color 0 . Let $e$ and $e^{\prime}$ be distinct edges of $Q_{k}$, and let $M\left(e, e^{\prime}\right)$ be the set of edges that are at equal distance from $e$ and $e^{\prime}$. Note that $r(e)=r\left(e^{\prime}\right)$ if and only if $C \subset M\left(e, e^{\prime}\right)$. Letting $m$ be the number of edges of $Q_{k}$, we have

$$
\operatorname{Prob}\left[r(e)=r\left(e^{\prime}\right)\right]=\left(\begin{array}{c}
\left|M\left(e, e^{\prime}\right)\right| \\
c
\end{array}\right) /\left(\begin{array}{c}
m \\
c
\end{array}\right) \leq\left(\left|M\left(e, e^{\prime}\right)\right| / m\right)^{c} .
$$


Let $\rho$ denote the probability that some two edges have the same distance vector; it suffices to show that $\rho<1$. Choosing $c$ large enough to make the sum of $\operatorname{Prob}\left[r(e)=r\left(e^{\prime}\right)\right]$ over pairs $\left(e, e^{\prime}\right)$ be less then 1 would require $c>k$, which is not small enough. To improve this, we show that there are many pairs $\left(e, e^{\prime}\right)$ for which $M\left(e, e^{\prime}\right)$ is the same. If $\mathcal{M}_{d}=\left\{M\left(e, e^{\prime}\right): d\left(e, e^{\prime}\right)=d\right\}$, then

$$
\rho \leq \sum_{d=1}^{k} \sum_{M \in \mathcal{M}_{d}} \operatorname{Prob}[C \subset M] \leq \sum_{d=1}^{k} \sum_{M \in \mathcal{M}_{d}}(|M| / m)^{c}
$$

and it suffices to show that the latter expression tends to zero.

First we show that $\left|\mathcal{M}_{d}\right|$ is small. Given edges $e$ and $e^{\prime}$, let $r$ and $r^{\prime}$ be the indices such that $e_{r}=*=e_{r^{\prime}}$. Let $i$ be an index such that $e_{i}=e_{i}^{\prime} \neq *$. Let $f$ and $f^{\prime}$ be obtained from $e$ and $e^{\prime}$, respectively, by changing only the $i$ th position, so $f_{i}=f_{i}^{\prime}=1-e_{i}$. We show that $M\left(e, e^{\prime}\right) \subseteq M\left(f, f^{\prime}\right)$, and hence by symmetry $M\left(e, e^{\prime}\right)=M\left(f, f^{\prime}\right)$. For $g \in M\left(e, e^{\prime}\right)$, we have

$$
d(f, g)=d(e, g)+\delta=d\left(e^{\prime}, g\right)+\delta=d\left(f^{\prime}, g\right), \quad \text { where } \quad \delta=\left\{\begin{aligned}
0 & \text { if } g_{i}=* \\
1 & \text { if } g_{i}=e_{i} \\
-1 & \text { if } g_{i}=1-e_{i}
\end{aligned}\right.
$$

We have shown that the set $M\left(e, e^{\prime}\right)$ does not depend on the values in the $i$ th positions of $e$ and $e^{\prime}$ when $e_{i}=e_{i}^{\prime} \neq *$. If $d\left(e, e^{\prime}\right)=d$, then $M\left(e, e^{\prime}\right)$ is therefore determined by $r, r^{\prime}$, and the values of $e$ in the $d-1$ positions $i$ such that $\left\{e_{i}, e_{i}^{\prime}\right\}=\{0,1\}$. We can specify $r$ and $r^{\prime}$ in $k^{2}$ ways, we can complete $e$ and $e^{\prime}$ in positions $r$ and $r^{\prime}$ in one way or four ways (depending on whether $r=r^{\prime}$ ), we can choose the locations of the differences in $\left(\begin{array}{l}k-1 \\ d-1\end{array}\right)$ ways or $\left(\begin{array}{l}k-2 \\ d-1\end{array}\right)$ ways (depending on whether $r=r^{\prime}$ ), and then we can complete $e$ in those positions in $2^{d-1}$ ways. To simplify the bound, note that $\left(\begin{array}{l}k-1 \\ d-1\end{array}\right) 2^{d-1}$ counts the lists of length $k-1$ from $\{0,1,2\}$ with $d-1$ nonzero positions. Therefore,

$$
\left|\mathcal{M}_{d}\right| \leq 4 k^{2}\left(\begin{array}{l}
k-1 \\
d-1
\end{array}\right) 2^{d-1}<4 k^{2} 3^{k-1}
$$

We will also need two bounds on $|M|$ for $M \in \mathcal{M}_{d}$ :

$$
\begin{aligned}
& |M| \leq m / 2, \text { and } \\
& |M| \leq k 2^{k-d+1}\left(\begin{array}{c}
d-1 \\
\lfloor(d-1) / 2\rfloor
\end{array}\right) .
\end{aligned}
$$

To prove the first inequality, suppose first that $e$ and $e^{\prime}$ differ in only one position. In that position one must have 0 and the other 1 , and then $M$ is precisely the set of edges whose $k$-tuples have $*$ in that position. Thus $|M|=2^{k-1}<m / 2$.

Otherwise, $e$ and $e^{\prime}$ differ in positions $i$ and $j$ with $i<j$. In this case, we construct an injection $\phi: M \rightarrow E\left(Q_{k}\right)-M$. Consider an arbitrary edge $f=$ $\left(f_{1}, \ldots, f_{k}\right)$. If $f_{i} \neq *$, then let $\phi(f)$ be the edge obtained by changing $f_{i}$ to $1-f_{i}$ and leaving the rest of the $k$-tuple unchanged. If $f_{i}=*$, then change $f_{j}$ to $1-f_{j}$ instead. The map is injective, and its image is outside $M$ because $\phi(f)$ is closer than $f$ to exactly one of $\left\{e, e^{\prime}\right\}$, regardless of the location of $*$ in $e$ or $e^{\prime}$. 
We now prove the second inequality. Let $d=d\left(e, e^{\prime}\right)$, and let $S=\left\{i:\left\{e_{i}, e_{i}^{\prime}\right\}=\right.$ $\{0,1\}\}$; we have observed that $|S|=d-1$. To construct an edge $f \in M\left(e, e^{\prime}\right)$, we first place its $*$ in one of $k$ positions and next assign values in positions outside $S$ in one of at most $2^{k-d+1}$ ways (one position may already have $*$ ).

The partially constructed $f$ may have some positions that contribute to distance from $e$ and $e^{\prime}$. Since these positions are outside $S$, the contributions are almost the same; the total may differ by 1 due to the positions where $e$ and $e^{\prime}$ have $*$. The values in the positions within $S$ (other than the possible $*$ ) must yield $f \in M\left(e, e^{\prime}\right)$. Thus at this stage there is a fixed $s$ such that $f$ must agree with $e$ in $s$ positions of $S$ and with $e^{\prime}$ in the remaining unspecified positions of $S$. These positions can be chosen in at most $\left(\begin{array}{c}d-1 \\ s\end{array}\right)$ ways, and this is bounded by $\left(\begin{array}{c}d-1 \\ \lfloor(d-1) / 2\rfloor\end{array}\right)$. The product of the bounds on the number of choices for each stage in the construction of $f$ yields the second inequality.

Armed with these inequalities, we bound $\rho$. Since $\left(\begin{array}{c}n \\ n / 2\end{array}\right) \sim 2^{n} / \sqrt{\pi n / 2}$, we have $\left(\begin{array}{c}d-1 \\ \lfloor(d-1) / 2\rfloor\end{array}\right) \leq \sqrt{2} \cdot 2^{d-1} / \sqrt{\pi(d-1) / 2}$ when $d$ is sufficiently large (actually, $d \geq 2$ ). Our final computation implies the existence of a coloring that establishes $\operatorname{dec}\left(Q_{k}\right) \leq$ $c+1$. We choose $d_{0}=k / \lg ^{2} k$ to break the sum into two parts.

$$
\begin{aligned}
\rho & \leq \sum_{1 \leq d \leq d_{0}} \sum_{M \in \mathcal{M}_{d}}(|M| / m)^{c}+\sum_{d_{0}<d \leq k} \sum_{M \in \mathcal{M}_{d}}(|M| / m)^{c} \\
& \leq \sum_{1 \leq d \leq d_{0}} k^{2}\left(\begin{array}{l}
k-1 \\
d-1
\end{array}\right) 2^{d+1}\left(\frac{m / 2}{m}\right)^{c}+\sum_{d_{0}<d \leq k} 4 k^{2} 3^{k}\left(\frac{k 2^{k-d+1}\left(\begin{array}{c}
d-1 \\
\lfloor(d-1) / 2\rfloor
\end{array}\right)}{k 2^{k-1}}\right)^{c} \\
& \leq d_{0} k^{d_{0}+1} 2^{d_{0}+1-c}+\sum_{d_{0}<d \leq k} 4 k^{2} 3^{k}(4 / \sqrt{\pi(d-1)})^{c} \\
& \leq 2^{\lg k-2 \lg \lg k+k / \lg k+\lg k+k / \lg ^{2} k+1-3.17 k / \lg k}+4 k^{3} 3^{k}\left(4 / \sqrt{\pi d_{0}}\right)^{c} \\
& \leq 2^{-1.17 k / \lg k+2 \lg k}+2^{2+3 \lg k+k \lg 3+(3.17 k / \lg k)\left(2-0.5 \lg \pi-0.5 \lg \left(k / \lg ^{2} k\right)\right)} .
\end{aligned}
$$

The last expression tends to 0 ; it suffices to choose $k$ large enough to make it less than 1.

\section{Cartesian Products}

The family of cartesian products of identical complete graphs contains all complete graphs and hypercubes. W. Imrich asked whether this family is well-behaved for decomposition dimension; we explore this question.

The cartesian product $G \square G^{\prime}$ has vertex set $V(G) \times V\left(G^{\prime}\right)$. Vertices $\left(u, u^{\prime}\right)$ and $\left(v, v^{\prime}\right)$ are adjacent in $G \square G^{\prime}$ if and only if $(1) u^{\prime}=v^{\prime}$ and $u v \in E(G)$ or $(2) u=v$ and $u^{\prime} v^{\prime} \in E\left(G^{\prime}\right)$. Thus we can view edges in $G \square G^{\prime}$ as consisting of an edge from one of the graphs and a vertex from the other graph.

Let $G^{k}$ denote the $k$ th cartesian power $G^{k}=G \square G \square \cdots \square G$. If $G$ has $n$ vertices and diameter $d$, then $G^{k}$ has $k e(G) n^{k-1}$ edges and diameter $k d$. When $G=K_{n}$, we have $e(G)=\left(\begin{array}{l}n \\ 2\end{array}\right)$ and $d=1$. This yields roughly $\operatorname{dec}\left(K_{n}^{k}\right) \geq(k+1) \lg n / \lg (k+1)$, which reduces to roughly the familiar values when $k=1$ or $n=2$.

Definition 6.1. A coloring of the edges of $G$ is totally distinguishing if

(1) the resulting distance vectors of edges are distinct, and

(2) the sets of colors incident to vertices are also distinct. 
For example, the coloring we gave to establish the upper bound for $\operatorname{dec}\left(K_{n}\right)$ is totally distinguishing, but the coloring we gave for $Q_{k}$ is not. Since $Q_{k}$ has $2^{k}$ vertices and we use only $\Theta(k / \lg k)$ colors, there are not enough different subsets of colors to distinguish the vertices.

Definition 6.2. Given edge-colorings $c$ and $c^{\prime}$ of $G$ and $G^{\prime}$, the product coloring $c^{*}$ of $E\left(G \square G^{\prime}\right)$ is defined by $c^{*}\left(\left(e, v^{\prime}\right)\right)=c(e)$ and $c^{*}\left(\left(v, e^{\prime}\right)\right)=c^{\prime}\left(e^{\prime}\right)$.

Theorem 6.3. If $c$ and $c^{\prime}$ are totally distinguishing edge-colorings for $G$ and $G^{\prime}$ using disjoint color sets, then the product coloring $c^{*} i s$ totally distinguishing for $G_{1} \square G_{2}$.

Proof. If two edges have the same distance vector under $c^{*}$, then they must have the same color under $c^{*}$. Since the colors in the product coloring are split according to which factor generates an edge, we may therefore restrict our attention to edges of the form $\left(e, u^{\prime}\right)$ and $\left(f, v^{\prime}\right)$. Suppose that $r\left(\left(e, u^{\prime}\right)\right)=r\left(\left(f, v^{\prime}\right)\right)$.

Split the coordinates of the distance vectors into two sets corresponding to the colors used in $G$ and $G^{\prime}$. Since the structure of the product coloring respects the factors, the coordinates corresponding to $c$ tell us that $r\left(e, u^{\prime}\right)=r\left(f, v^{\prime}\right)$ requires $r(e)=r(f)$ under $c$, and hence $e=f$. Also, the coordinates corresponding to $c^{\prime}$ that have value 1 in $r\left(e, u^{\prime}\right)$ are the colors incident to $u^{\prime}$ under $c^{\prime}$. Since $c^{\prime}$ is totally distinguishing, we conclude that $r\left(e, u^{\prime}\right)=r\left(f, v^{\prime}\right)$ also requires $u^{\prime}=v^{\prime}$. Hence the product coloring generates distinct distance vectors.

Since the set of colors incident to a vertex $\left(u, u^{\prime}\right)$ of $G \square G^{\prime}$ under $c^{*}$ is the union of the sets incident to $u$ under $c$ and to $u^{\prime}$ under $c^{\prime}$, it follows also that the sets of colors incident to distinct vertices of $G \square G^{\prime}$ under $c^{*}$ are distinct. Hence $c^{*}$ is totally distinguishing.

Corollary 6.4. If $G$ has a totally distinguishing coloring with c colors, then $\operatorname{dec}\left(G^{k}\right) \leq k c$.

Corollary 6.5. $\operatorname{dec}\left(K_{n}^{k}\right)=\Theta(\lg n)$ for fixed $k$.

Thus the family of cartesian products of identical cliques with a fixed number of factors is well-behaved. When $n$ is fixed and $k$ is large, this method does not work, because the factor $k$ is too large to yield the desired bound. For small fixed $n$, it may be possible to extend the method used in the proof of Theorem 6.3.

\section{ACKNOWLEDGEMENT}

The authors thank Noga Alon for clarifying and strengthening the results in Sections 3 and 4 . Thanks also go to a referee for repeated careful readings and suggestions.

\section{REFERENCES}

[1] B. Bollobás, Random Graphs, Academic Press, London, 1985 (1st ed.), Cambridge University Press, Cambridge, 2001 (2nd ed.).

[2] G. Chartrand, D. Erwin, M. Raines, and P. Zhang, The decomposition dimension of graphs, Graphs Combin. 17 (2001), 599-605.

[3] D. Coppersmith and J. B. Shearer, New bounds for union-free families of sets, Electronic Journal of Combinatorics 5 (1998), \#R39.

[4] H. Enomoto, Upper bound of the decomposition dimension of a graph, manuscript. 
[5] H. Enomoto and T. Nakamigawa, On the decomposition dimension of trees, manuscript.

[6] F. Harary and M. Plantholt, Graphs and applications (Boulder, Colo., 1982), Wiley, New York, 1985 , pp. 147-162.

[7] S. Janson, T. Łuczak, and A. Ruciński, Random Graphs, Wiley-Interscience, New York.

[8] T. Nakamigawa, A note on the decomposition dimension of complete graphs, manuscript.

[9] J. Petersen, Die Theorie der regulären Graphen, Acta Math. 15, 193-220.

Faculty of Environmental Information, Keio University 5322 Endoh, Fujisawa, KANAGAWA 252-8520, JAPAN

E-mail address: hagita@sfc.keio.ac.jp

Department of Mathematics, California State University, San Marcos CA 92096

E-mail address: akundgen@csusm.edu

Department of Mathematics, University of Illinois, Urbana, Illinois 61801

E-mail address: west@math.uiuc.edu 